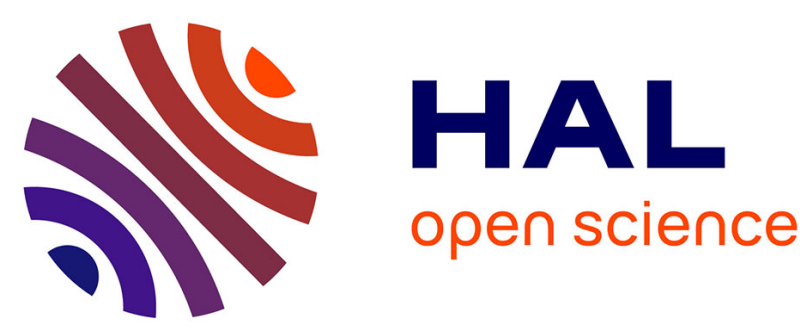

\title{
PtxtPME1 and homogalacturonans influence xylem hydraulic properties in Poplar
}

Thierry Allario, Aude Tixier, Hosam Awad, Cédric Lemaire, Nicole

Brunel-Michac, Eric Badel, Tete Severien Barigah, Jean-Louis J.-L. Julien,

Pierre Peyret, Ewa J. Mellerowicz, et al.

\section{To cite this version:}

Thierry Allario, Aude Tixier, Hosam Awad, Cédric Lemaire, Nicole Brunel-Michac, et al.. PtxtPME1 and homogalacturonans influence xylem hydraulic properties in Poplar. Physiologia Plantarum, 2018, 163 (4), 31 p. 10.1111/ppl.12702 . hal-01728105

\section{HAL Id: hal-01728105 \\ https://hal.science/hal-01728105}

Submitted on 15 Oct 2020

HAL is a multi-disciplinary open access archive for the deposit and dissemination of scientific research documents, whether they are published or not. The documents may come from teaching and research institutions in France or abroad, or from public or private research centers.
L'archive ouverte pluridisciplinaire HAL, est destinée au dépôt et à la diffusion de documents scientifiques de niveau recherche, publiés ou non, émanant des établissements d'enseignement et de recherche français ou étrangers, des laboratoires publics ou privés. 


\section{PtxtPME1 and homogalacturonans influence xylem hydraulic properties in poplar}

Thierry Allario a,b , Aude Tixier ${ }^{a}$, Hosam Awad ${ }^{c}$, Cedric Lemaire $^{a}$, Nicole Brunel $^{a}$, Eric Badel ${ }^{a}$, Têtè S. Barigaha ${ }^{a}$, Jean-Louis Julien ${ }^{a}$, Pierre Peyret ${ }^{b}$, Ewa J. Mellerowicz ${ }^{d}$, Herve Cochard ${ }^{a}$ and Stephane Herbette ${ }^{a, *}$ (D)

aUCA, INRA, PIAF, 63000 Clermont-Ferrand, France

bUCA, EA 4678 CIDAM, 63000 Clermont-Ferrand, France

'Agriculture and Botany Department, Faculty of Agriculture, Menoufia University, Shebin El-Kom, Egypt

${ }^{d}$ Department of Forest Genetics and Plant Physiology, Swedish University of Agricultural Sciences, 90183 Umeå, Sweden

\section{Correspondence \\ ${ }^{*}$ Corresponding author, \\ e-mail: stephane.herbette@uca.fr}

Received 21 December 2017;

revised 15 January 2018

doi:10.1111/ppl.12702
While the xylem hydraulic properties, such as vulnerability to cavitation (VC), are of paramount importance in drought resistance, their genetic determinants remain unexplored. There is evidence that pectins and their methylation pattern are involved, but the detail of their involvement and the corresponding genes need to be clarified. We analyzed the hydraulic properties of the 35S::PME1 transgenic aspen that ectopically under- or over-express a xylem-abundant pectin methyl esterase, PtxtPME1. We also produced and analyzed $4 C L 1:: P G / l$ transgenic poplars expressing a fungal polygalacturonase, AnPGII, under the control of the Ptxa4CL1 promoter that is active in the developing xylem after xylem cell expansion. Both the 35S::PME1 under- and over-expressing aspen lines developed xylem with lower-specific hydraulic conductivity and lower VC, while the 4CL1::PGIl plants developed xylem with a higher VC. These xylem hydraulic changes were associated with modifications in xylem structure or in intervessel pit structure that can result in changes in mechanical behavior of the pit membrane. This study shows that homogalacturonans and their methylation pattern influence xylem hydraulic properties, through its effect on xylem cell expansion and on intervessel pit properties and it show a role for PtxtPME1 in the xylem hydraulic properties.

\section{Introduction}

According to the cohesion-tension theory, sap flows from root to shoot in vascular plants through xylem conduits under negative pressure (Tyree 1997, Steudle 2001). This negative pressure makes the liquid sap metastable, with a risk of vaporization by cavitation inside conduits that considerably increase in plants during drought. Cavitation makes the conduits air-filled or embolized, and they can no longer transport water. During extreme drought, extensive embolism blocks water transport leading to organ or plant death (Barigah et al. 2013). The resistance to drought-induced embolism is thus considered as an important adaptive trait for the woody species (Brodribb and Cochard 2009, Choat et al. 2012, Lens et al. 2013). A global analysis on the relationship between vulnerability to cavitation (VC) and drought resistance pointed out the narrow hydraulic safety margin at which trees commonly operate (Choat et al. 2012), inferring that research is needed on xylem VC under the current

Abbreviations - 4CL, 4-(hydroxy)cinnamoyl CoA ligase; AFM, atomic force microscopy; HG, homogalacturonan; PEMs, pectin methylesterases; PLC, percentage loss of xylem conductance; PM, pit membrane; RH, relative humidity; VC, vulnerability to cavitation; WAK, wall associated kinases. 
climate changes. Yet the genetic determinants of this critical trait are poorly understood.

In angiosperm species, sap flows through xylem vessels, which are formed by the perforated xylem vessel elements. To move from one vessel to another, the sap must flow via pits in the secondary cell wall. These pits represent an important limiting factor for the rate of water flow in the xylem (Wheeler et al. 2005, Choat et al. 2006), but they are also barriers that block air passage between vessels (Lens et al. 2013). This ability of pits to prevent the passage of air bubbles is of key importance for VC. Indeed, drought-induced cavitation occurs as a result of air seeding through the pit membrane (PM) separating a sap-filled vessel under tension from an air-filled vessel at atmospheric pressure (Cochard 2006). The VC is determined by several quantitative and qualitative pit characteristics, but their respective contribution is still in debate (Choat and Pittermann 2009, Lens et al. 2013), which hinders the research on the genetic basis for VC. Considering variability in the porosity of PM and the occurrence of air-seeding in the largest PM pore, the probability of air-seeding into a conduit would increase in a stochastic fashion with its pit area (Jarbeau et al. 1995, Wheeler et al. 2005, Christman et al. 2009). This "rare pit" hypothesis is supported by a positive correlation between VC and the average area of pit connecting vessels based on average VC and pit area from around 80 species (Wheeler et al. 2005, Hacke et al. 2006). The vessel pitting is expected to be greater in xylem with short, highly connected and grouped vessels than in xylem with long and solitary vessels, supporting a role for the three-dimensional arrangement and connectivity of vessels in VC (Choat and Pittermann 2009). The "rare pit" hypothesis is not supported by other studies, suggesting the importance of qualitative pit features (Lens et al. 2011, Scholz et al. 2013). Within angiosperms, a huge interspecific variation in the PM thickness and porosity exists, and the relationship between these two parameters indicates that thicker PMs have smaller pores and are more resistant to air-seeding (Jansen et al. 2009). The VC also depends on the mechanical behavior of the PM, especially its resistance to stretching that relies on the pit geometry (Choat et al. 2004, 2008, Sperry and Hacke 2004, Tixier et al. 2014). PM porosity and mechanical properties are also tributary to their biochemistry, but we know little about this, despite recent insights on the biochemical composition (Herbette et al. 2015).

In poplars, the intervessel PM is composed of cellulose microfibrils and contains epitopes of lignins, whereas pectins epitopes are only concentrated in the annulus, i.e. the border area of the PM (Plavcova and Hacke 2011, Kim and Daniel 2013, Herbette et al. 2015). Homogalacturonans (HGs), which are the most abundant polymers of pectins, can be demethylesterified at the C- 6 carboxyl (Wolf et al. 2009) to various degrees by pectin methylesterases (PMEs) (Pelloux et al. 2007). When HGs are demethylesterified in blocks, intermolecular $\mathrm{Ca}^{2+}$ bonds are formed between pectins chains. As a result, the pectin mesh forms pectin gels that strengthen pectin structure, which impacts the mechanical and porosity properties of the wall matrix (Willats et al. 2001). Although pectins epitopes are restricted to the edge of PM, larger pores for air seeding are frequently found at the periphery of the PM (Hillabrand et al. 2016) and our previous studies argue for a critical role of pectins and their methylesterification pattern in VC (Herbette and Cochard 2010, Dusotoit-Coucaud et al. 2014). Branches perfused with pectins hydrolyzing enzymes or with $\mathrm{Ca}^{2+}$ chelators showed an increased VC. We hypothesized that the role of calcium in VC may be dependent on the HG content in the PM and its methylesterification pattern, and consequently on PME activity. By creating $\mathrm{Ca}^{2+}$ bonds, PME activity would strengthen the pectin meshwork in the PM annulus. This would in turn protect pectins from hydrolysis during pit maturation, reduce pore sizes in PM and increase the annulus resistance to PM stretching. Moreover, PME activity is involved in mechanisms determining the cell dimensions in developing xylem (Siedlecka et al. 2008), and could thus affect xylem organization. We hypothesize that PME could monitor the three-dimensional arrangement and connectivity of vessels and thus the xylem hydraulic properties (Choat and Pittermann 2009). Hence, PMEs appear as good candidates for mediating hydraulic properties of xylem at different levels.

Plant functional genomics approach is a powerful tool that permits to characterize biological function of particular genes and to highlight gene interactions in biological processes. In this study, using transgenic aspen and poplar lines, we investigated the role of $\mathrm{HG}$ in the hydraulic properties of the PM and the role of a $P M E$ gene in the xylem hydraulic traits that relies both on the xylem vessel properties and on the pit properties. For the latter objective, we used the aspen transgenic lines that were either over-expressing or under-expressing PtxtPME1 under the 35SCAMV promoter (35S::PME1, Siedlecka et al. 2008) and that were thus affected in HG methylesterification. For the former objective, we constructed the poplar transgenic lines overexpressing AnPGll gene coding for polygalacturonase (PG; E.C.4.2.2.2) (Capodicasa et al. 2004), which cleaves the $\alpha-1,4$-glycosidic bond between the galacturonic acid units of HG, thus that were affected in HG chain length. To specifically target pit primary cell wall and avoid effects on xylem cell expansion, we expressed AnPGIl under the control of the Ptxa4CL1 promoter. Populus 
4CL1 is a gene encoding 4-(hydroxy)cinnamoyl CoA ligase $(4 \mathrm{CL})$ involved in monolignol biosynthesis, which is expressed specifically in developing xylem after xylem cell expansion (Lu et al. 2004).

\section{Materials and methods}

\section{Plant material and culture conditions}

The study was performed on transgenic Populus lines modified for expression of genes involved in pectin metabolism. Transgenic lines ectopically over-expressing (line 2B and 7) or under-expressing (by co-supppression, line 5) PtxtPME1 fused with 35S CAMV promoter were generated in Populus tremula $\times$ tremuloides clone T89 (Siedlecka et al. 2008). The generation of the transgenic lines overexpressing the fungal polygalacturonase AnPGIl fused the xylem-specific Ptxa4CL1 promoter (Lu et al. 2004) in Populus tremula $\times$ alba clone 717-1B4 is described in a further separate section.

All plants were multiplied in vitro on Murashige and Skoog medium. At $4 \mathrm{~cm}$ of height, plants were gradually acclimatized in hydroponic solution and grown in a climatic chamber with $16 \mathrm{~h}$ daylight $\left(100 \mu \mathrm{mol} \mathrm{m} \mathrm{m}^{-2} \mathrm{~s}^{-1}\right)$ at $22 \pm 1{ }^{\circ} \mathrm{C}$ (day) and $19 \pm 1{ }^{\circ} \mathrm{C}$ (night) with $70 \pm 10 \%$ of relative humidity $(\mathrm{RH})$. At $30 \mathrm{~cm}$ of height, plants were potted into 10-I pots filled with a commercial soil (40\% black, 30\% brown and 30\% blond peat moss, $\mathrm{pH} 6.1$, DUMONA-RN 75-3851 Arandon, Holland) and grown for at least 90 days in the greenhouse with $16 \mathrm{~h}$ daylight $\left(350 \mu \mathrm{mol} \mathrm{m} \mathrm{m}^{-2} \mathrm{~s}^{-1}\right)$ at $22 \pm 1^{\circ} \mathrm{C}$ (day) and $19 \pm 1^{\circ} \mathrm{C}$ (night) with $60 \pm 20 \% \mathrm{RH}$. Water supply by dripping was controlled to get between 60 and $100 \%$ of field capacity.

We also used xylem material from Populus tremula $x$ alba (clone 717-1B4) plants grown in 10 I pots under three contrasting water regimes, in a previous study (Awad et al. 2010). A 10-cm long segment was collected above the one used for analysis of VC. This xylem material was used to investigate the expression of PtxaPME1.

\section{RNA extraction and CDNA synthesis}

Xylem tissue was isolated by peeling the bark. The xylem core was then immediately frozen in liquid nitrogen and stored at $-80^{\circ} \mathrm{C}$. Tissue was ground in liquid nitrogen and $2 \mathrm{~g}$ of ground xylem from each plant were pooled for RNA extraction. RNA was isolated using CTAB (Cetyl Trimethyl Ammonium Bromide) extraction buffer as described by Chang et al. (1993) and was treated with DNase I. First strand cDNAs were synthesized using the Super Script ${ }^{\mathrm{TM}}$ III first strand synthesis system (Invitrogen, Paisley, UK) with an oligo-dT according to the manufacturer's instructions.

\section{Generation of 4CL1::PGII poplar lines}

The AnPG/l cDNA sequence was previously fused with the sequence encoding a signal peptide of PGIP1 from Phaseolus vulgaris, and the resulting fused sequence was cloned in pBI121 (Capodicasa et al. 2004). We amplified the gene fusion from a preparation of this construct using the primers 5'-ATGACTCAATTCAATATCCCAG-3' and 5'-TAACAAGAGGCCACCGAA-3'. The Ptxa4CL1 promoter sequence was amplified from genomic DNA of Populus tremula $\times$ alba using the primers $5^{\prime}$-GACG GTGGCATGAACACAAAGC-3' and 5'-TGGCGAGTTTT AGGTGCTCT-3' given in Lu et al. (2004). Finally, the polymerase chain reaction (PCR) products were inserted into PGEM-Teasy vector (Promega, Madison, WS), transformed into $\mathrm{DH} 5 \alpha$ Escherichia coli strain, and they were sequenced on both strands.

PGIP1-AnPGII and Ptxa4CL1 promoter sequences were cloned into gateway vectors (Gateway ${ }^{\circledR}$ Cloning, Invitrogen, Carlsbad, CA). Entry clones, resulting from the recombination of AnPGIl or Ptxa4CL1 promoter sequences in donor vectors, were transfected into $E$. coli by heat shock. Transformed E. coli (One Shot ${ }^{\circledR}$ Mach1 ${ }^{\mathrm{TM}}$ T1 R, ThermoFisher, Waltham, MA) were grown on selective medium with kanamycin and targeted gene incorporation was verified by PCR and sequencing. Entry clone with Ptxa4CL1 promoter and entry clone containing PGIP1:AnPG/l hybrid cDNA sequence were recombined in pK7WG destination vector. The recombined pK7WG vector was transferred to poplar by Agrobacterium-mediated transformation as described in Leple et al. (1992). Kanamycin-resistant lines were selected and clonally propagated in vitro. Transgene insertions were checked in selected lines by PCR as described by Mai et al. (2010).

\section{Expression analysis}

Expression levels of PtxtPME1 were assessed by quantitative real time PCR (qPCR) in xylem from poplar plants grown under three contrasting water regimes (Awad et al. 2010). The single strand cDNA was used as a template in qPCR as described in Mai et al. (2010). qPCRs were carried out in a iCycler iQ ${ }^{\mathrm{TM}}$ (Bio-Rad, Hercules, CA), using platinum Taq DNA polymerase (Invitrogen, Paisley, UK), SYBR Green (Sigma, St. Louis, MO; 1:1000 in 25\% dimethyl sulfoxide), and the primers $5^{\prime}$-GAGATGGTTAGGGAGG-3' and 5'-AATTACAGTTCGCCGAG-3'. The program was as follows: $95^{\circ} \mathrm{C}$ for $5 \mathrm{~min}, 40$ cycles of $94^{\circ} \mathrm{C}$ for $15 \mathrm{~s}, 55^{\circ} \mathrm{C}$ for $20 \mathrm{~s}$ and $72^{\circ} \mathrm{C}$ for $30 \mathrm{~s}$, followed by a melting curve analysis to check the specificity of fragment amplification. The cDNA expression was normalized according to $18 S$ rRNA expression (Nualpun et al. 2005). The method 
to calculate the relative expression ratio is described by Pfaffl (2001).

Expression of AnPGIl was checked by semiquantitative RT-PCR in 4CL1::PG/l lines and WT plants grown for 2 months in the greenhouse in order to allow wood to be developed. PCR was performed on the first strand CDNA with the primers 5'-ATGACTCAATTCAATATCCCAG-3' and 5'-AGTAGAG CATTTCGCCTTGC-3'. The program was as follows: $95^{\circ} \mathrm{C}$ for $2 \mathrm{~min}, 30 \mathrm{cycles}$ of $94^{\circ} \mathrm{C}$ for $30 \mathrm{~s}, 60^{\circ} \mathrm{C}$ for $20 \mathrm{~s}$ and $72^{\circ} \mathrm{C}$ for $30 \mathrm{~s}$. To check for similar quantity of cDNA, we also amplified the $18 \mathrm{~S}$ rRNA cDNA as a control with the same conditions.

\section{Leaf water potential and growth measurements}

Midday leaf water potential $\left(\Psi_{\mathrm{md}}\right)$ was measured for each plant on two mature leaves per plant with a pressure bomb (PMS instrument, Albany, OR) at solar noon just before harvesting plants for hydraulic measurements. Plant size and stem diameter were measured, respectively, with a meter tape and a caliper.

\section{Native embolism and specific conductivity}

Entire stem of each plant was defoliated, harvested, wrapped in moist paper, sealed in humid black plastic bag and stored at $4^{\circ} \mathrm{C}$ for hydraulic measurements at the laboratory (within 2 days). Native embolism and specific conductivity were measured using XYL'EM ${ }^{\circledR}$ (Bronkhorst, Montigny-les-Cormeilles, France), as described by Herbette and Cochard (2010). The xylem hydraulic conductivity was measured on $0.35 \mathrm{~m}$ long stem segments prepared from the upper part of the plant. We checked that there was no open vessel at both ends of segments using the low pressure air injection test (Ewers and Fisher 1989). Then, we measured the xylem area on cross sections prepared from both ends. The xylem-specific conductivity of the sample was determined by dividing the hydraulic conductivity by the mean xylem area, taking then into consideration differences in xylem cross-sectional area.

\section{Vulnerability curves}

Vulnerability to xylem cavitation was measured on $0.38 \mathrm{~m}$ long stem segments located just below the part used for specific conductivity measurement using the Cavitron technique (Cochard 2002, Cochard et al. 2005). Four to eight samples, each from a different individual tree, were measured for each transgenic line. The technique uses a centrifugal force that increases the water tension in the xylem segment, and the resulting loss of hydraulic conductance is measured at the same time. The curve of percentage loss of xylem conductance (PLC) vs xylem water tension represents the sample's vulnerability curve. Xylem pressure $(P)$ was first set to a reference pressure $(-0.5 \mathrm{MPa})$ and the maximal conductance $\left(K_{\max }\right)$ of the sample was determined. The xylem pressure was then set to a more negative value for $3 \mathrm{~min}$ and the conductance $(K)$ of the sample was remeasured. The PLC of the sample was then computed as:

$$
\mathrm{PLC}=100 \times\left(1-K / K_{\max }\right)
$$

The procedure was repeated for more negative pressures (with -0.25 or -0.5 MPa step increments) until PLC reached at least $90 \%$. The PLC curves were fitted using the following sigmoid function:

$$
\operatorname{PLC}=100 /\left(1+\mathrm{e}^{S(P-P 50) / 25}\right)
$$

where $P_{50}$ is the xylem pressure causing $50 \%$ loss of conductance and $s$ is the slope of the curve at this point (Pammenter and Van Der Willigen 1998).

To test for the role of calcium in VC in transgenic plants, stem segments were infiltrated a $10 \mathrm{mM}$ sodium phosphate solution at $\mathrm{pH} 10$ as described in Herbette and Cochard (2010) before Cavitron analyses.

\section{Light and transmission electron microscopy}

For light and electron microscopy, we only used fresh samples to avoid any modification of the PM structure as reported (Li et al. 2016, Zhang et al. 2017). Samples were prepared below the basal part of stem segments that were used to measure VC, as described in Herbette et al. (2015).

\section{Light microscopy}

Semi-thin $(2 \mu \mathrm{m})$ cross sections were stained with polychromatic Toluidine blue dye and were observed with an optical microscope (Zeiss Axioplan 2, Zeiss, Jena, Germany). Pictures were taken and analyzed using IMAGE) software (Schneider et al. 2012). Vessels were isolated by automatic segmentation and we measured their frequency $\left(F_{\mathrm{v}}\right.$, number of vessel per wood area, $\left.\mathrm{mm}^{-2}\right)$, their diameters $\left(D_{\mathrm{v}}, \mu \mathrm{m}\right)$, the fraction of wood area they occupied (the total lumen area per wood area, $\left.A_{v}\right)$, the solitary vessel index $\left(S_{v}\right.$, ratio of total number of solitary vessels to total vessel groupings) and the contact fraction $\left(F_{\mathrm{C}}=\right.$ fraction of the total vessel wall perimeter in contact with another vessel). Analyses were performed on four plants per line, each plant represented by 33 to 64 cross sections for vessels parameters. 
Table 1. List of parameters used in modeling the PM mechanical behavior with symbols, units, definitions and equations.

\begin{tabular}{|c|c|c|c|c|}
\hline & Symbol & Unit & Definition & Equation \\
\hline \multirow[t]{2}{*}{ Constants } & $E$ & $\mathrm{~Pa}$ & Young's modulus of PM & \\
\hline & V & - & Poisson's ratio $=0.3$ & \\
\hline \multirow[t]{4}{*}{ Input variables } & $D_{\mathrm{p}}$ & $\mu \mathrm{m}$ & Pit membrane diameter & \\
\hline & $L_{p}$ & $\mu \mathrm{m}$ & $\begin{array}{l}\text { Distance from the PM surface to the } \\
\text { inner pit aperture }\end{array}$ & \\
\hline & $D_{\mathrm{a}}$ & $\mu \mathrm{m}$ & Pit aperture diameter & \\
\hline & $T_{\mathrm{m}}$ & $\mu \mathrm{m}$ & PM thickness & \\
\hline \multirow[t]{3}{*}{ Output variables } & $D$ & $\mathrm{MPa} \mathrm{nm^{3 }}$ & PM flexural rigidity & $D=\left(E^{*} T_{\mathrm{m}}{ }^{3}\right) / 12\left(1-v^{2}\right)$ \\
\hline & $P_{\mathrm{b}}$ & $\mathrm{MPa}$ & $\begin{array}{l}\text { Pressure required to deflect the } \\
\text { membrane against the aperture }\end{array}$ & $P_{b}=1 / 3^{*} E /\left(1-v^{2}\right)^{*} T_{m}{ }^{3} /\left(D_{p}{ }^{2}-D_{a}{ }^{2}\right)^{2 *} L_{p}$ \\
\hline & $\epsilon$ & - & Maximum PM strain at $P_{\mathrm{b}}$ & $\varepsilon_{\max P b}=\varepsilon(r=0)=-(3 / 8) *\left(\left[1-v^{2}\right] / E\right) *\left(R^{2} / T_{\mathrm{m}}{ }^{2}\right) * P_{\mathrm{b}}$ \\
\hline
\end{tabular}

\section{Transmission electron microscopy}

Ultra-thin $(70 \mathrm{~nm})$ cross sections were prepared and observed as described in Dusotoit-Coucaud et al. (2014). Aperture length $\left(D_{\mathrm{a}}\right)$, pit chamber depth $\left(L_{\mathrm{p}}\right)$, pit diameter $\left(D_{\mathrm{p}}\right)$ and PM thickness $\left(T_{\mathrm{m}}\right)$ were measured as described in Tixier et al. (2014), Table 1, Fig. 1). These measurements were performed on images with pits showing two apertures, and neither shrinkage nor stretching of the PM as these modifications can disturb the PM structure ( $\mathrm{Li}$ et al. 2016, Zhang et al. 2017). On the same images, the primary wall thickness $\left(T_{\mathrm{pw}}\right)$ was measured between adjacent vessels, near the pits. Analyses were performed on four plants per line, each plant represented by 27 to 66 pits for pit parameters.

\section{Mechanical model of PM deflection}

The flexural rigidity of the membrane $(D)$, the pressure needed to deflect the membrane against the pit aperture $\left(P_{\mathrm{b}}\right)$ and the mechanical strain of the PM beyond the aspiration pressure $\left(\varepsilon_{\mathrm{b}}\right)$ were estimated using the mechanical model developed and described in Tixier et al. (2014). Input and output variables of this model are listed in Table 1.

\section{Results}

\section{Expression of 4CL1::PGII in poplar increases VC}

Five 4CL1::PG/l transgenic lines (3.1, 5.1, 10.1, 11.1 and 12.1) showed an AnPG/l expression in xylem tissues by RT-PCR (Fig. 2). Line 10.1 exhibited the highest relative transcript levels, lines 3.1 and 11.1 were intermediate, whereas lines 12.1 and 5.1 were the lowest expressing lines. Although the Populus 4CL1 promoter offers a developmental control of over-expression, which is limited to late stages of xylogenesis, this restriction makes the detection of enzymatic activity in the protein extract impossible (Lu et al. 2004).
No differences in height growth, leaf water potential, native embolism or specific hydraulic conductivity between 4CL1::PG/l lines and wild-type (WT) plants were observed; however, xylem pressure causing 50\% of loss of hydraulic conductance $\left(P_{50}\right)$ was significantly higher (less negative) in the transgenic lines (Table 2). Xylem vulnerability curves that were used to calculate $P_{50}$ are presented in Fig. 3B. These curves clearly show that the transgenic lines exhibit significantly higher loss of conductance over a large spectrum of xylem pressures than the WT plants. This indicates that the expression of polygalacturonase during late stages of xylogenesis caused an increase in xylem VC.

\section{Xylem hydraulic traits in 35S::PME1 transgenic lines}

To investigate the role of PME in the hydraulic properties of xylem, we used the 35S::PME1 expressing aspen (lines 7, 2B and 5). Unlike the 4CL1::PGI/ expressing plants, the over- or under-expression of PtxtPME1 induced a reduction in height growth (Table 2). Leaf water potential and native embolism was not altered in transgenic plants when compared to WT plants (Table 2). However, the specific hydraulic conductivities were lower in transgenic lines both over- and under-expressing PtxtPME1 than in WT plants (Table 2). Moreover, stems from 35S::PME1 lines $2 \mathrm{~B}$ and 5 were significantly less vulnerable to cavitation than WT ( $P_{50}$ values in Table 2$)$. Line 7 showed intermediate $P_{50}$ values (Table 2), which is consistent with a lower change in the degree of methylesterification in this line and in the xylem structure (Siedlecka et al. 2008). The lower vulnerability of the 35S::PME1 lines compared to WT can be clearly seen by analyzing the $P_{50}$ values and the vulnerability curves over an entire spectrum of xylem pressure (Table 2, Fig. 3A). Thus, with regard to the xylem vulnerability, 35S::PME1 and 4CL1::PG/l transgenic lines behave in the opposite way, the former is less vulnerable, while the latter is more vulnerable to hydraulic loss under water 


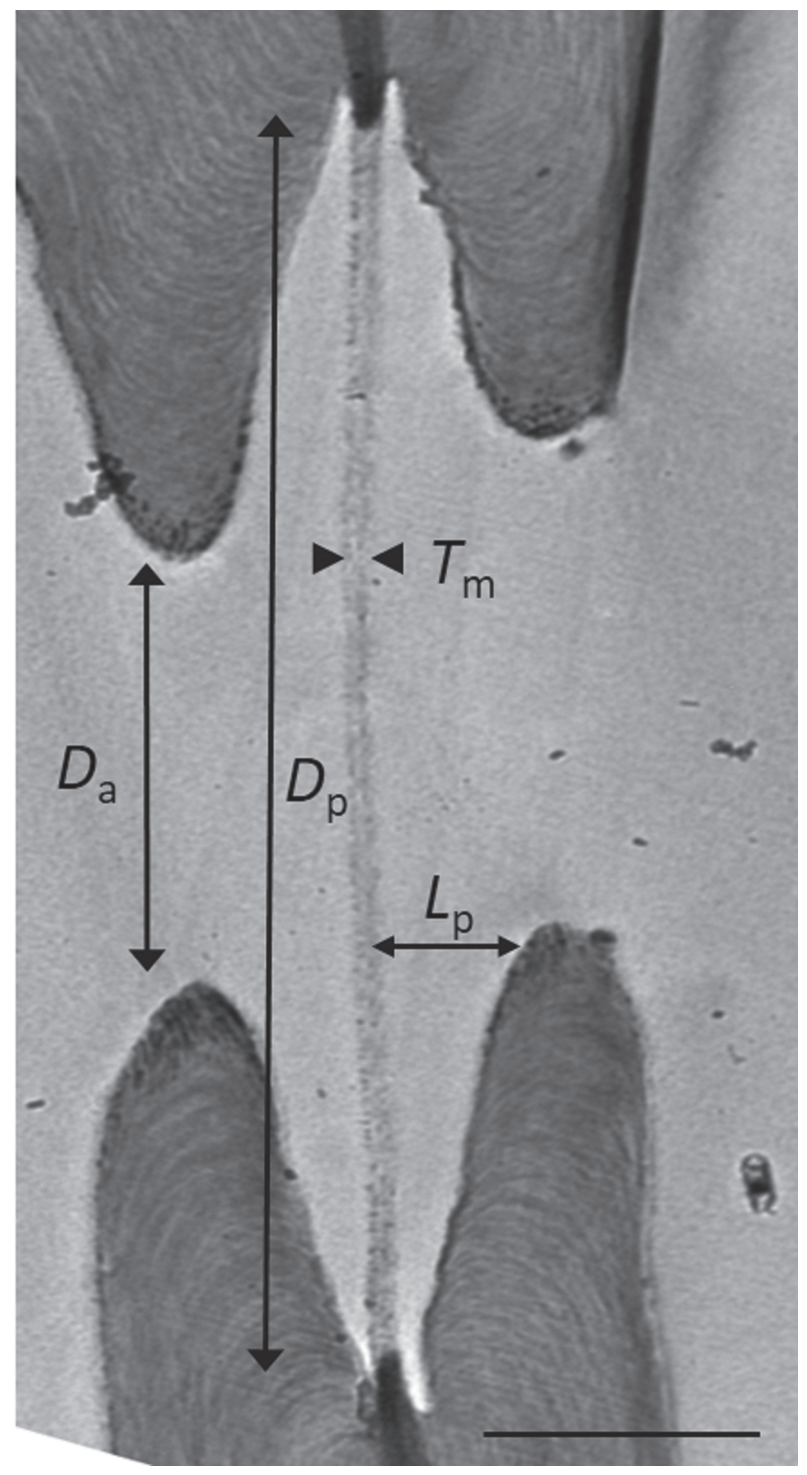

Fig. 1. A transverse section of a bordered pit as observed using TEM. Black arrows show the measured pit dimensions (Table 1): $D_{a}$, pit aperture diameter; $D_{p}$, pit membrane diameter; $L_{p}$, pit chamber depth; $T_{m}$, pit membrane thickness. Scale bar $=2 \mu \mathrm{m}$.

stress. The low levels in native embolism and lack of difference between the transgenic lines and the respective WT (Table 2) exclude an effect of the native embolism on the VC measurements. If a set of plants have higher level of embolism, then the vulnerability curves would be plotted on the remaining functional vessels, which are the most resistant, resulting in an artificial decrease in VC. It was thus essential to observe similar levels of native embolism across genotypes in order to decipher true differences from artificial shifts in vulnerability curves. To test for the role of pectin-bound calcium in the transgenic lines, the stem segments were infiltrated
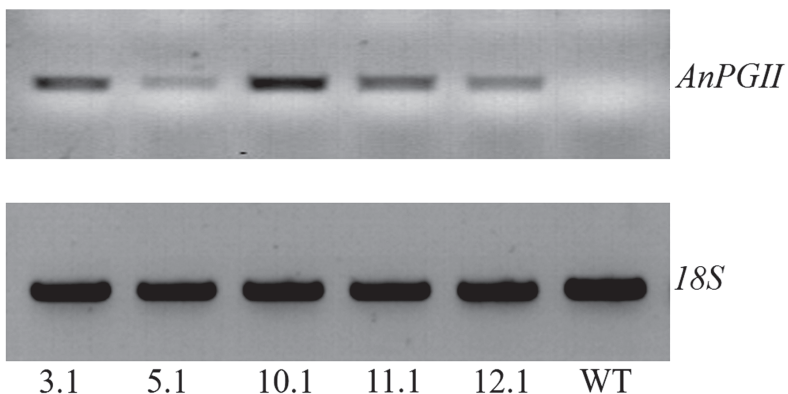

Fig. 2. Expression of $A n P G / /$ in the $4 C L 1:: P G / /$ transgenic plants. Transcript accumulation was analyzed by RT-PCR as described in the section Materials and methods, then the PCR products were separated on agarose gels. The name of the line is indicated below each lane, and the name of the target gene is indicated to the right of the panel. 18S rRNA was used as the loading control. This result is representative of three replicates.

with a sodium phosphate solution that removes calcium from the wall before Cavitron analyses (Fig. 4). No modification was observed for $P_{50}$ values between treated and mock-treated branches in each tested line, suggesting that the calcium removal did not affect their VC.

Although this was not the objective of this study, and 4CL1::PGIll lines and 35S::PME1 lines cannot be compared since they were grown and analyzed separately, their respective WT plants showed difference for their VC (Fig. 3) especially for their $P_{50}$ values (Table 2).

\section{Xylem structural traits in 35S::PME1 and 4CL1::PGII transgenic lines}

To understand how PtxtPME1 and AnPG/l could modify the xylem hydraulic traits, we investigated several anatomical traits of the xylem and the pit structures in the respective transgenic lines (Table 3 ). We found a relationship between PtxtPME1 expression level and vessel frequency $\left(F_{\mathrm{v}}\right)$ or vessel diameter $\left(D_{\mathrm{v}}\right)$. Indeed, the vessel density increased with PtxtPME1 expression whereas the mean vessel diameter followed an opposite trend. In addition, line 5 had higher solitary vessel index $\left(S_{v}\right)$ and lower contact fraction $\left(F_{\mathrm{c}}\right)$ compared to other lines. In contrast, the anatomical parameters of the vessels of the $4 C L 1:: P C / l$ lines were not significantly different from WT.

Investigations on the primary wall thickness and on the intervessel pit structure using transmission electron microscopy analysis were carried out on the transgenic lines that displayed the greatest difference for $P_{50}$ with WT (35S::PME1 - lines 2B and 5 and 4CL1::PGII line 5.1). First, the line 5 showed a thinner primary wall compared to WT (Table 3), while no difference was observed for the other lines. At the pit level, there 
were differences between $355:: P M E 1$ and $4 C L 1:: P G / I$ lines and WT plants for all parameters measured: $D_{\mathrm{p}^{\prime}}$ $L_{\mathrm{p}}, D_{\mathrm{a}}$ and $T_{\mathrm{m}}$ (Table 3). The lines $2 \mathrm{~B}$ and 5 showed smaller $D_{p}$, smaller $L_{p}$ but larger $D_{\mathrm{a}}$ and $T_{\mathrm{m}}$. The line 5.1 showed bigger pits than WT plants, with significantly higher values for every parameter measured.

To evaluate the impact of these structural pit modifications, the PM mechanical behavior was estimated using these structural parameters and the mechanical model developed by Tixier et al. (2014). The maximum PM strain $\left(\varepsilon_{\max }\right)$ as a function of pressure was different between the 35S::PME1 lines and WT plants, especially with a lower slope of strain for the line 2B (Fig. 5A). The behavior of line 5 was opposite. Thus, the PM was stiffer in the line overexpressing PME1 and less stiff in the line with suppressed PME1 expression, compared to WT. In contrast to these pronounced effects in the 35S::PME1 lines, the mechanical behavior of PM in line 5.1 overexpressing 4CL1::PGIl was not affected (Fig. 5B), because the overall pit relative geometry was not altered in this line compared to WT despite the overall increase in size of the pit parameters (Table 3). Important PM mechanical parameters such as experienced strains for a given pressure and pressure required to deflect the PM against pit borders $\left(P_{\mathrm{b}}\right)$ were similar (Fig. $\left.5 \mathrm{~B}\right)$.

\section{Expression of PME1 in poplars grown under contrasted water regimes}

Since expression of PME1 affected several key variables related to xylem hydraulic conductivity in transgenic plants, we were asking if PME1 could have a role in determining xylem hydraulic properties under drought in relation to the level of acclimation to water stress. Therefore, we subjected WT poplar to different water regimes, which resulted in differences in their VC (Awad et al. 2010), and analyzed the expression level of PtxaPME1 in the xylem of these plants. The PtxaPME1 expression was found to be reduced in poplars grown under drier conditions and displaying lower VC (Fig. 6).

\section{Discussion}

We conducted two distinct experiments to investigate the role of HGs in PM hydraulics and the role of a PME gene in the hydraulic properties of the xylem. The respective WT for the 4CL::PGII and 35S::PME1 transgenic lines show differences for $P_{50}$ (Table 2) and for some structural traits of xylem vessels or pits (Table 3). This can be explained by their different genetic backgrounds (Cochard et al. 2007), and by different growth conditions as the VC has been reported to depend on growth conditions (Harvey and Van Den Driessche 1999, Awad et al. 

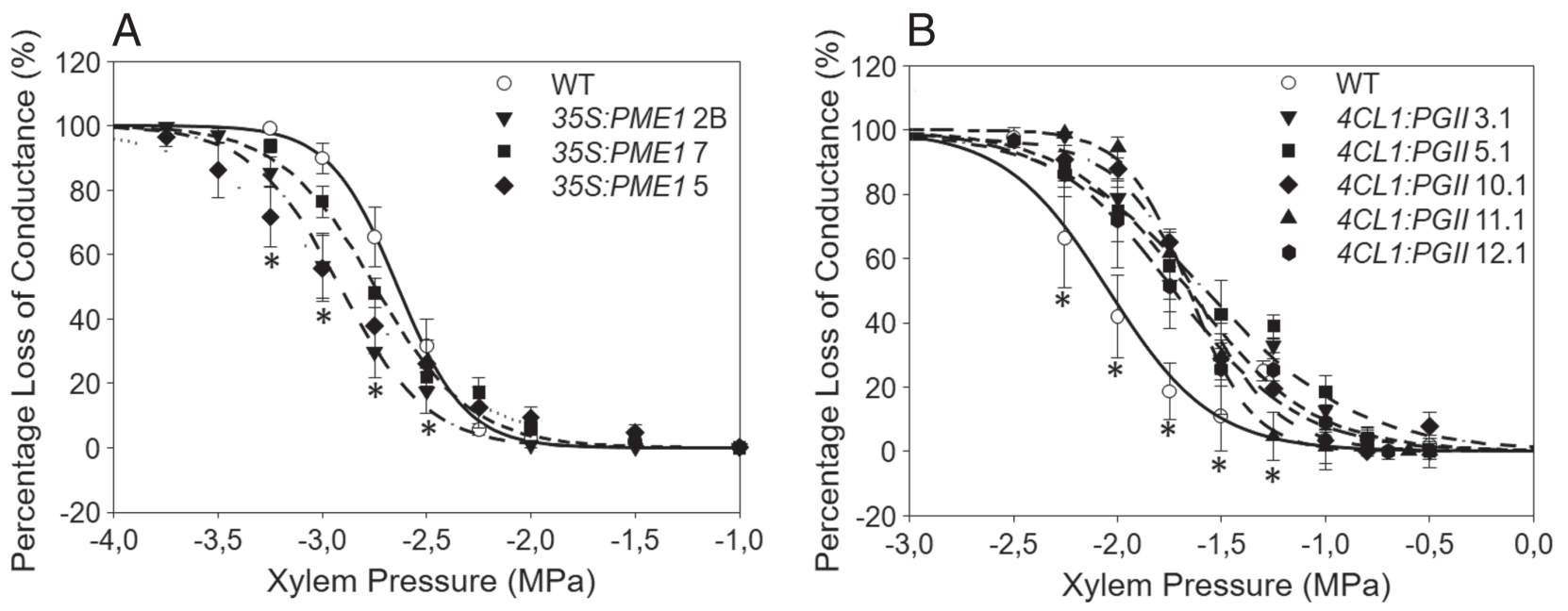

Fig. 3. Xylem vulnerability curves of stems from the transgenic plants expressing $355:: P M E 1$ (A) or $4 C L 1:: P G / l(B)$. The percentage loss of conductance with decreasing xylem water potential is represented for the 35S::PME1 and 4CL1::PGIl lines (closed symbols) and their corresponding WT plants (T89 and 717-1B4, respectively, open symbols). Curves represent the fitted data according to equation from Pammenter and Van Der Willigen (1998, see section materials and methods). Each data point represents the mean value from five to nine samples and bars represent standard errors. For each pressure point, values from transgenic plants significantly different from wild-type plants are marked of an asterisk (Student's $t$-test, $P<0.05$ ).

2010, Herbette et al. 2010). The trees were grown in greenhouse, which does not allow an exact reproducibility of conditions between distinct experiments. Such differences between the respective WT did not allow comparing the 35S::PME1 lines with the 4CL::PGIl lines.

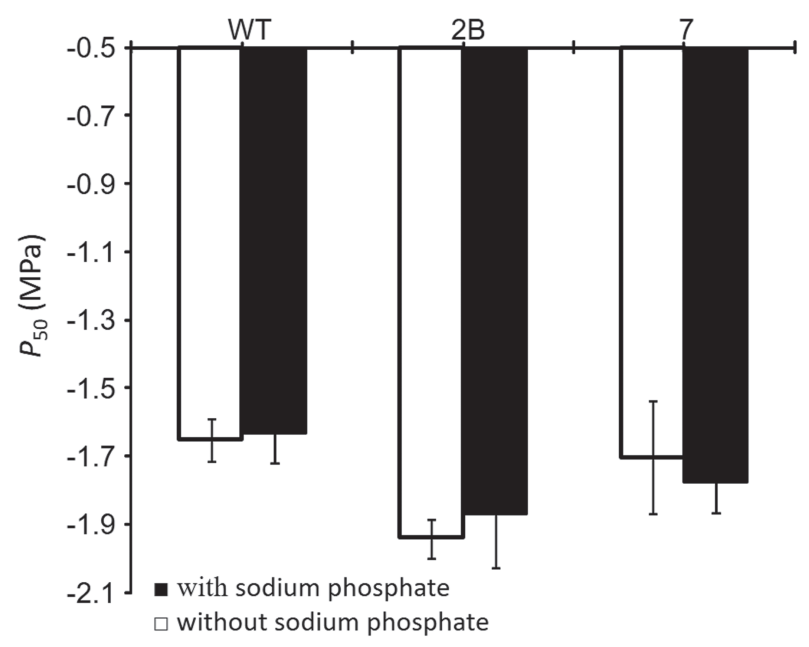

Fig. 4. Effect of calcium removal on the xylem VC of stems from the transgenic plants expressing 35S::PME1. The xylem pressure inducing $50 \%$ of PLC $\left(P_{50}\right)$ is deduced from vulnerability curves of stems perfused $(\square)$ or not perfused $(\square)$ with a sodium phosphate solution chelating calcium. The analyses were performed on the transgenic lines $2 \mathrm{~B}$ and 7 and on the WT plants (T89). Data are mean values from three to nine samples and bars represent standard errors. No significant difference was found between treatments in each genotype (Student's $t$-test, $P<0.05$ ).

\section{Role of pectins in PM hydraulic properties}

Hydraulic conductivity is a complex trait that is of key importance for plant survival and productivity. Here, we attempted to dissect some of the underlying mechanisms involved in regulation of this trait, using transgenic plants. 4CL::PG/l transgenics allowed questioning the importance of HGs in the PM for hydraulic conductivity without its confounding effects on xylem cell growth. The results demonstrated the importance of intact HG in the PM - the transgenic lines all exhibited significant increases vulnerability when fungal polygalacturonase is expressed in fully grown xylem cells.

Investigating the role of $\mathrm{HG}$ in PM is a tricky task since the pectin epitopes were only detected in the annulus, a restricted area of the PM (Plavcova and Hacke 2011, Herbette et al. 2015). When infiltrating the stems with a sodium phosphate solution that precipitates calcium from the wall (Herbette and Cochard 2010), the VC of the lines WT and 2B was not modified as compared to mock-treated stems (Fig. 4), suggesting that pectin-bound calcium does not influence the PM hydraulic properties. This is not surprising since calcium removal did not affect the VC for other hygrophilous species such as Betula and Salix (Herbette and Cochard 2010). Experiments with stem infiltration with a pectin-hydrolyzing enzyme support a role for pectins in VC (Dusotoit-Coucaud et al. 2014), but there was no evidence that it was the pectins located in the PM. Here, the greater VC in $4 C L 1:: P G / l$ plants support a role in VC for PM-located pectins. Many changes were observed in their pit structure (Table 3), but they did not 


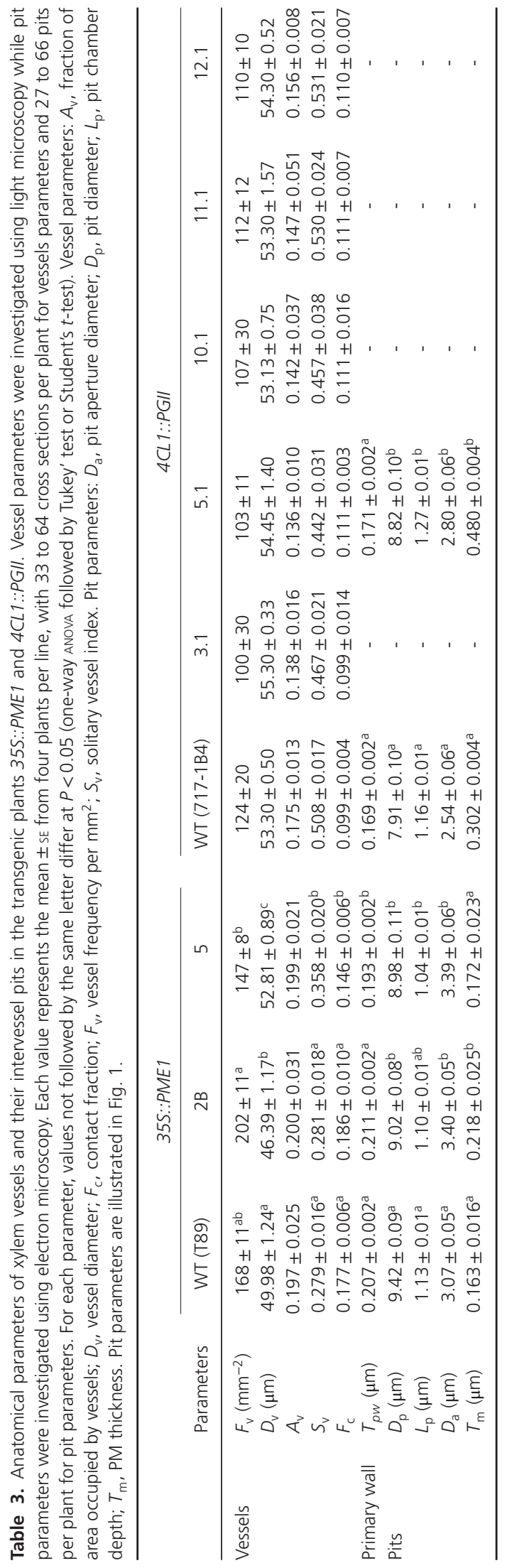

result in a change in mechanical behavior of the PM as modeled from pit structure (Fig. 5). We assume that AnPGIl partly hydrolyzed the pectins that are highly concentrated in the PM annulus (Herbette et al. 2015). Although the role of this annulus is unclear, it probably ensures the mechanical resistance of PM to strain (Plavcova and Hacke 2011, Herbette et al. 2015). Thus, weakening the PM by pectin hydrolysis would increase the VC. Moreover, it could also increase PM porosity in the region of annulus which would increase air-seeding. Unfortunately, measuring the PM porosity is a tricky task, especially for investigating the effect of pectin hydrolysis on pore size. A major concern in such analyses using electron microscopy is the alteration of ultrastructural features during sample preparation (Choat et al. 2003, Pesacreta et al. 2005, Jansen et al. 2008). For scanning or transmission electronic microscopy, sample preparation involves dehydration of tissue (replacing water with ethanol then with resin) that alters subtle features such as pit porosity and the highly hydrated pectin network. Such preparation artifacts would explain the discrepancy between the observed and calculated pore size for air seeding (Jansen et al. 2009). In addition, pits observed under electron microscopy are in a relaxed state, whereas PM pores enlarge by stretching when they undergo a pressure difference across conduits (Choat et al. 2004, Sperry and Hacke 2004, Tixier et al. 2014). A solution to these methodological problems is provided by a study employing atomic force microscopy (AFM), in which PMs were examined in non-dried and dried states (Pesacreta et al. 2005). However, this technique is not suitable for measurement series on PMs because it is difficult to access this tiny structure.

\section{Role of PME1 in xylem hydraulic properties}

Previous studies indicated that PME1 is a dominant PME in developing wood, expressed mainly in the early and late xylem cell expansion phase, and mediating xylem cell expansion (Hertzberg et al. 2001, Pelloux et al. 2007, Siedlecka et al. 2008). Here, we report that PtxtPME1 mRNA accumulation is reduced in xylem formed under dry conditions and with a low VC (Fig. 6). An under-expression of PtxtPME1 in transgenic plants also induced a lower xylem VC (Fig. 3). These results suggest that PtxtPME1 might indeed be involved in regulation of wood hydraulic properties and that the action of PME1 can be important at many levels: anatomy, chemistry and mechanical properties of PM, and PM porosity. Since the VC is determined by several quantitative and qualitative structural traits, there are many putative ways for PtxtPME1 to influence the wood hydraulic properties. Besides, under-expression and ectopic over-expression 
A

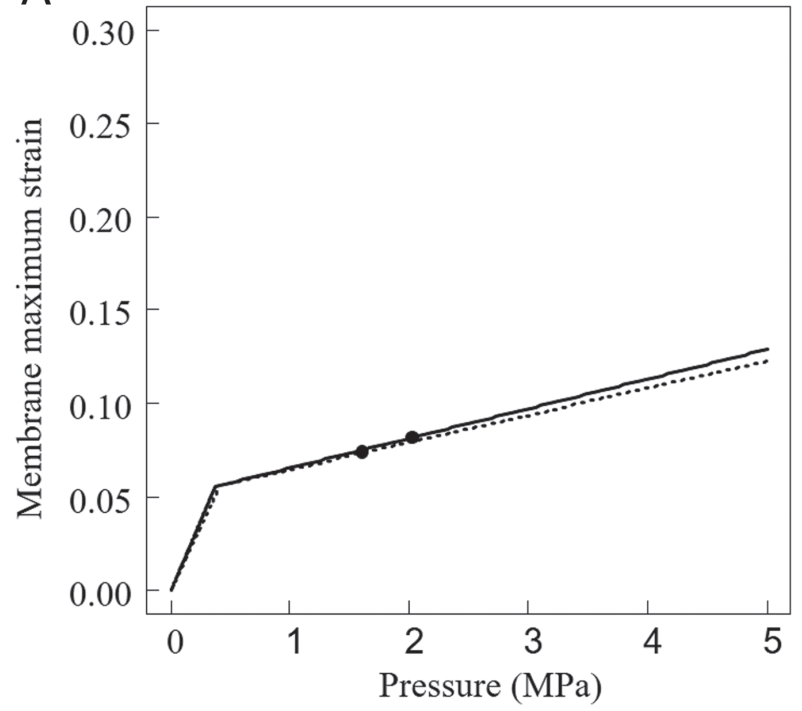

B

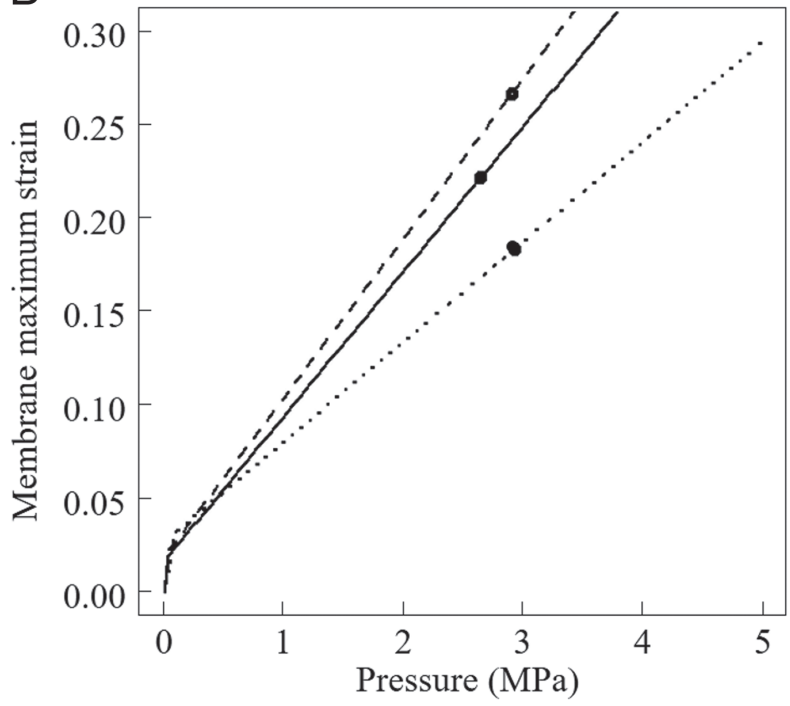

Fig. 5. Maximum PM strain $\left(\varepsilon_{\max }\right)$ plotted against pressure for the transgenic plants expressing $355:: P M E 1$ (A) or $4 C L 1:: P G / l(B)$. The $\varepsilon_{\text {max }}$ was calculated using input values of pit parameters measured in Table 3 for the lines expressing 35S::PME1 (line 2B and 5, dotted and dashed lines, respectively), the line $4 C L 1: \because P G / / 5.1$ (dotted line) and their respective WT plants (T89 and 717-1B4, respectively, continuous line). Black dots represent the value of membrane maximum strain reached for $P_{50}$ mean value in each line.

of PtxtPME1 induced different structural modifications that were associated with decrease in VC and specific hydraulic conductivity (Table 2).

What happens to the primary cell wall when the methylester pattern changes are not obvious for explaining the effect on hydraulic properties in 35S::PME1

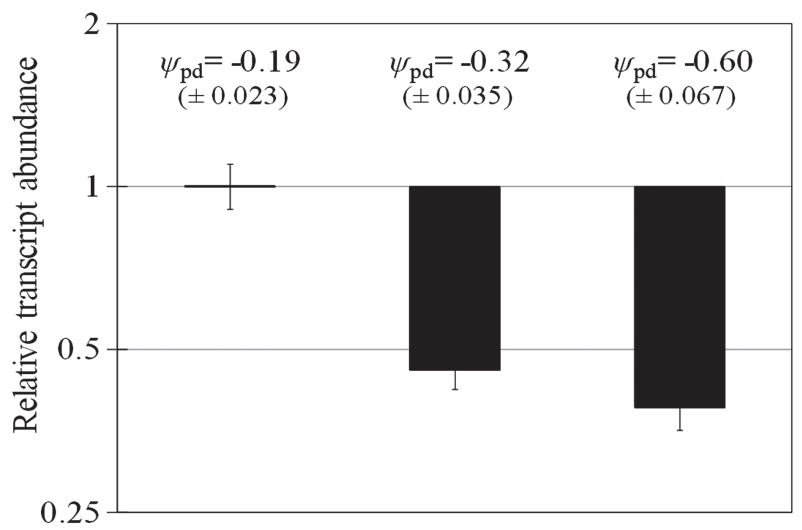

Fig. 6. RT-qPCR analysis of the relative abundance of the PtxaPME1 transcripts under different water regimes in WT plants. Relative transcript abundance was expressed as the ratio of gene expression level in moderately water stressed ( $\psi_{\text {pd }}=-0.32$ ) or severe water-stressed plants $\left(\psi_{\mathrm{pd}}=-0.60\right)$ compared with plants grown under optimal water regime $\left(\psi_{\mathrm{pd}}=-0.19\right)$. Data are means values from three replicates and bars represent standard deviations. For each water condition, mean values of predawn water potentials $\left(\psi_{\text {pd }}\right.$ ) measured on plants prior to RT-qPCR analysis were indicated, with standard deviations in brackets. transgenic poplars. Only the line 5 showed a weak reduction of the primary wall thickness, but this did not result in a reduction of the PM thickness (Table 3) since the opposite trend was observed. The molecular mechanisms occurring in 35S::PME1 transgenic poplars are not known, but previous studies put the light on a role for HG methylesterification in a wall-dependent cell signaling that regulate developmental processes, especially through wall-associated kinases (WAK) pathway (Kohorn 2016). Considering this role, we thus propose a functional understanding of the relationship between the PtxtPME1 expression level and the xylem hydraulic properties. For the xylem of the line $2 \mathrm{~B}$, the decrease in the specific hydraulic conductivity can be related to the vessels' diameter. Such vessel diameter reduction in the line 2B agrees with a role of PtxtPME1 in inhibiting cell growth of vessel elements (Siedlecka et al. 2008). The higher PME activity in the line $2 \mathrm{~B}$ was also shown to stabilize the pectin networks (Siedlecka et al. 2008). Consequently, the hydrolysis of the PM would be hindered during PM maturation leading to a thicker PM in the line 2B compared to WT plants. This thicker PM may contribute to both the decrease in conductivity, and to the decrease in VC, by increasing the pressure required for air-seeding (Jansen et al. 2009). This is consistent with the fact that a thicker PM drastically reduces the mechanical strain the PM experiences (Tixier et al. 2014). Increase in PtxtPME1 activity affects the overall pit geometry with changes in PM and aperture diameters $\left(D_{\mathrm{p}}\right.$ and $D_{\mathrm{a}}$, respectively). To 
test the impact of these changes in VC, these pit parameters have been integrated in the mechanical model proposed by Tixier et al. (2014). PM of the line 2B appeared to experience lower maximum strain than the PM of the WT at equal pressure, which may result in a decrease of VC, as highlighted between species (Tixier et al. 2014).

In the line 5, the lower PtxtPME1 expression level induced an increase in vessel diameter that suggests that the decrease in hydraulic conductivity is not related to an increase in lumen resistance, but rather to an increase in end wall resistance. First, the vessel grouping parameters, i.e. the contact fraction $\left(F_{\mathrm{c}}\right)$ and the solitary vessel index $\left(S_{v}\right)$, are significantly lower and higher in the line 5 compared to WT plants. These parameters quantify the actual overlap between neighboring vessels and thus allow estimating the vessel connectivity. $F_{\mathrm{c}}$ was shown to be strongly correlated to VC and hydraulic conductivity in a study carried out on Acer species (Lens et al. 2011), while $S_{v}$ was measured and found to be negatively correlated to VC across different Prunus species (Scholz et al. 2013). The changes in $F_{\mathrm{c}}$ and $S_{\mathrm{v}}$ would thus support the decreases in hydraulic conductivity and VC in the line 5. Moreover, the lower pit diameter $\left(D_{\mathrm{p}}\right)$ and pit chamber depth $\left(L_{p}\right)$ values in the line 5 , when compared to $\mathrm{WT}$, can also contribute in reducing the conductivity. The pit diameter is directly influencing the area allowing the sap flow between vessel, while $L_{p}$ influences the water flow inside the pit chamber (Sperry and Hacke 2004, Schulte et al. 2015). A recent study reported an oxidative stress and tylose formation in the wood of the line 5 (Lesniewska et al. 2017). Such ingrowths into the lumen of xylem vessels would contribute to the decrease hydraulic conductance in the line 5, but they cannot explain a lower VC. The oxidative stress in wood would also affect the wall metabolism, including the lignification process (Mader and Amberg-Fisher 1982), and would be an explanation for the structural changes in wood observed for this line.

In addition to providing support for the importance of HGs in PM for hydraulic conductivity, and identifying PME1 as a genetic component of hydraulic properties, our study contributes to the understanding of trades-off in xylem functions. We confirm the supposed role of structural traits in the trade-off between safe xylem (low $\mathrm{VC}$ ) and efficient xylem (high conductivity), especially $T_{\mathrm{m}}, F_{\mathrm{c}}$ and $L_{\mathrm{p}}$ (Lens et al. 2011).

While transgenic approach is a powerful mean to test for gene function, there are limitations due to strong and ectopic gene expression. In addition to the transgenic plants investigated here, we also used transgenic plants over-expressing PtxtPL1-27 (Biswal et al. 2014), a pectate lyase that degrades $\mathrm{HG}$, and is expressed at the onset of secondary wall formation during xylem differentiation. Despite a severe growth reduction, we did not observe changes in xylem hydraulic properties (Appendix S1), which can be explained by compensatory effects occurring early during xylogenesis. Previously, Arabidopsis polygalacturonase mutants were found to develop an inflorescence stem with a greater VC than WT plants (Tixier et al. 2013), highlighting a role for this gene and pectins in VC. However, how this gene affects the xylem hydraulic has not been clarified. We thus produced the $4 C L 1:: P G / /$ transgenic plants using the promoter Ptxa4CL1 that allows a late and a xylem-specific transgene expression. In addition to target the transgene expression, this expression pattern reduces the likelihood of inducing side effects with compensatory effects during xylogenesis. This is supported by a normal growth associated to changes in VC in 4C/1-PG/l plants. However, the AnPGIl expression is limited in time and space, and it is thus extremely challenging to detect increase in PG activity (Lu et al. 2004), that could weaken the effect on the phenotype. Untangling the genetic basis of $\mathrm{VC}$ is an extremely intricate process requiring meticulous genotyping and novel minute phenotyping techniques.

To conclude, our study demonstrates a role for HGs in the xylem hydraulic properties through their effect on the characteristics of vessels and intervessel pits, while the presence of pectins in PM and their contributions to hydraulic properties are debated in the literature. Moreover, it identifies the xylem-specific PME1 gene as a genetic component of the xylem hydraulic properties, and it puts in light the complexity of the genetic control of these hydraulic properties, as PME1 would influence them in different ways.

\section{Author contributions}

T.A., A.T. and H.A. performed most of the experiments, analyzed the data and wrote some parts of the manuscript; C.L. reviewed thoroughly the manuscript; N.B. contributed and supervised the microscope analyses; T.B. contributed and supervised the ecophysiological analyses; J.-L.J. and P.P. help in defining the research project; E.M. provided the PME transgenic plants and helped for the transgenic plant analyses; H.C. contributed in analyzing the ecophysiological data; S.H. supervised the whole research project, especially the students (A.T., T.A., C.L. and H.A.), and contributed to most of the analyses, and wrote most part of the paper.

Acknowledgements - The authors thank Christelle Boisselet, Pierre Conchon and Romain Souchal (PIAF laboratory) for their technical assistance, and Christelle Blavignac and Claire Szczepaniak from the CICS platform 
(Clermont-Ferrand, France) for running the TEM observations. We gratefully acknowledge the gift of the PGIP1 - AnPGIl fused construct from Professor Felice Cervone

\section{References}

Awad H, Barigah T, Badel E, Cochard H, Herbette S (2010) Poplar vulnerability to xylem cavitation acclimates to drier soil conditions. Physiol Plant 139: 280-288

Barigah TS, Charrier O, Douris M, Bonhomme M, Herbette S, Ameglio T, Fichot R, Brignolas F, Cochard H (2013) Water stress-induced xylem hydraulic failure is a causal factor of tree mortality in beech and poplar. Ann Bot 112: $1431-1437$

Biswal AK, Soeno K, Gandla ML, Immerzeel P, Pattathil S, Lucenius J, Serimaa R, Hahn MG, Moritz T, Jonsson LJ, Israelsson-Nordstrom M, Mellerowicz EJ (2014) Aspen pectate lyase PtxtPL1-27 mobilizes matrix polysaccharides from woody tissues and improves saccharification yield. Biotechnol Biofuels 7: 11

Brodribb TJ, Cochard H (2009) Hydraulic failure defines the recovery and point of death in water-stressed conifers. Plant Physiol 149: 575-584

Capodicasa C, Vairo D, Zabotina O, McCartney L, Caprari C, Mattei B, Manfredini C, Aracri B, Benen J, Knox JP, De Lorenzo G, Cervone F (2004) Targeted modification of homogalacturonan by transgenic expression of a fungal polygalacturonase alters plant growth. Plant Physiol 135: 1294-1304

Chang C, Kwok SF, Bleecker AB, Meyerowitz EM (1993) Arabidopsis ethylene-response gene ETR1. Similarity of product to two-component regulators. Science 262: 539-544

Choat B, Pittermann J (2009) New insights into bordered pit structure and cavitation resistance in angiosperms and conifers. New Phytol 182: 557-560

Choat B, Ball M, Luly J, Holtum J (2003) Pit membrane porosity and water stress-induced cavitation in four co-existing dry rainforest tree species. Plant Physiol 131: $41-48$

Choat B, Jansen S, Zwieniecki MA, Smets E, Holbrook NM (2004) Changes in pit membrane porosity due to deflection and stretching: the role of vestured pits. J Exp Bot 55: 1569-1575

Choat B, Brodie TW, Cobb AR, Zwieniecki MA, Holbrook NM (2006) Direct measurements of intervessel pit membrane hydraulic resistance in two angiosperm tree species. Am J Bot 93: 993-1000

Choat B, Cobb AR, Jansen S (2008) Structure and function of bordered pits: new discoveries and impacts on whole-plant hydraulic function. New Phytol 177: 608-625

Choat B, Jansen S, Brodribb TJ, Cochard H, Delzon S, Bhaskar R, Bucci SJ, Field TS, Gleason SM, Hacke UG, Jacobsen AL, Lens F, Maherali H, Martinez-Vilalta J,
Mayr S, Mencuccini M, Mitchell PJ, Nardini A, Pittermann J, Pratt RB, Sperry JS, Westoby M, Wright IJ, Zanne AE (2012) Global convergence in the vulnerability of forests to drought. Nature 491: 752-755

Christman MA, Sperry JS, Adler FR (2009) Testing the 'rare pit' hypothesis for xylem cavitation resistance in three species of Acer. New Phytol 182: 664-674

Cochard H (2002) A technique for measuring xylem hydraulic conductance under high negative pressures. Plant Cell Environ 25: 815-819

Cochard H (2006) Cavitation in trees. CR Physique 7: $1018-1126$

Cochard H, Damour G, Bodet C, Tharwat I, Poirier M, Ameglio T (2005) Evaluation of a new centrifuge technique for rapid generation of xylem vulnerability curves. Physiol Plant 124: 410-418

Cochard H, Casella E, Mencuccini M (2007) Xylem vulnerability to cavitation varies among poplar and willow clones and correlates with yield. Tree Physiol 27: $1761-1767$

Dusotoit-Coucaud A, Brunel N, Tixier A, Cochard H, Herbette $S$ (2014) Hydrolase treatments help unravel the function of intervessel pits in xylem hydraulics. Physiol Plant 150: 388-396

Ewers FW, Fisher JB (1989) Techniques for measuring vessel lengths and diameters in stems of woody plants. Am J Bot 86: 1077-1081

Hacke UG, Sperry JS, Wheeler JK, Castro L (2006) Scaling of angiosperm xylem structure with safety and efficiency. Tree Physiol 26: 689-701

Harvey HP, Van Den Driessche R (1999) Nitrogen and potassium effects on xylem cavitation and water-use efficiency in poplars. Tree Physiol 19: 943-950

Herbette S, Cochard H (2010) Calcium is a major determinant of xylem vulnerability to cavitation. Plant Physiol 153: 1932-1939

Herbette S, Wortemann R, Awad H, Huc R, Cochard H, Barigah TS (2010) Insights into xylem vulnerability to cavitation in Fagus sylvatica L.: phenotypic and environmental sources of variability. Tree Physiol 30: $1448-1455$

Herbette S, Bouchet B, Brunel N, Bonnin E, Cochard H, Guillon F (2015) Immunolabelling of intervessel pits for polysaccharides and lignin helps in understanding their hydraulic properties in Populus tremula x alba. Ann Bot 115: 187-199

Hertzberg M, Aspeborg H, Schrader J, Andersson A, Erlandsson R, Blomqvist K, Bhalerao R, Uhlen M, Teeri TT, Lundeberg J, Sundberg B, Nilsson P, Sandberg G (2001) A transcriptional roadmap to wood formation. Proc Natl Acad Sci USA 98: 14732-14737

Hillabrand RM, Hacke UG, Lieffers VJCPCER (2016) Drought-induced xylem pit membrane damage in aspen and balsam poplar. Plant Cell Environ 39: 2210-2220 
Jansen S, Pletsers A, Sano Y (2008) The effect of preparation techniques on Sem-imaging of pit membranes. IAWA J 29: 161-178

Jansen S, Choat B, Pletsers A (2009) Morphological variation of intervessel pit membranes and implications to xylem function in angiosperms. Am J Bot 96: 409-419

Jarbeau JA, Ewers FW, Davis SD (1995) The mechanism of water-stress-induced embolism in two species of chaparral shrubs. Plant Cell Environ 18: 189-196

Kim JS, Daniel G (2013) Developmental localization of homogalacturonan and xyloglucan epitopes in pit membranes varies between pit types in two poplar species. IAWA J 34: 245-262

Kohorn BD (2016) Cell wall-associated kinases and pectin perception. J Exp Bot 67: 489-494

Lens F, Sperry JS, Christman MA, Choat B, Rabaey D, Jansen S (2011) Testing hypotheses that link wood anatomy to cavitation resistance and hydraulic conductivity in the genus Acer. New Phytol 190: $709-723$

Lens F, Tixier A, Cochard H, Sperry JS, Jansen S, Herbette S (2013) Embolism resistance as a key mechanism to understand adaptive plant strategies. Curr Opin Plant Biol 16: 287-292

Leple JC, Brasileiro AC, Michel MF, Delmotte F, Jouanin L (1992) Transgenic poplars: expression of chimeric genes using four different constructs. Plant Cell Rep 11: $137-141$

Lesniewska J, Ohman D, Krzeslowska M, Kushwah S, Barciszewska-Pacak M, Kleczkowski LA, Sundberg B, Moritz T, Mellerowicz EJ (2017) Defense responses in aspen with altered pectin methylesterase activity reveal the hormonal inducers of tyloses. Plant Physiol 173: 1409-1419

Li S, Lens F, Espino S, Zohreh K, Klepsch M, Schenk HJ, Schmitt M, Schuldt B, Jansen S (2016) Intervessel pit membrane thickness as a key determinant of embolism resistance in angiosperm xylem. IAWA J 37: 152-171

Lu H, Zhao YL, Jiang XN (2004) Stable and specific expression of 4-coumarate:coenzyme a ligase gene (4CL1) driven by the xylem-specific Pto4CL1 promoter in the transgenic tobacco. Biotechnol Lett 26: 1147-1152

Mader M, Amberg-Fisher V (1982) Role of peroxidase in lignification of tobacco cells: I. Oxidation of nicotinamide adenine dinucleotide and formation of hydrogen peroxide by cell wall peroxidases. Plant Physiol 70: 1128-1131

Mai J, Herbette S, Vandame M, Cavaloc E, Julien JL, Ameglio T, Roeckel-Drevet P (2010) Contrasting strategies to cope with chilling stress among clones of a tropical tree, Hevea brasiliensis. Tree Physiol 30:

1391-1402
Nualpun S, Pluang S, Russell FD, Wallie S (2005) Molecular cloning of a new cDNA and expression of 3-hydroxy-3-menthylglutaryl-CoA synthase gene from Hevea brasiliensis. Planta 221: 502-512

Pammenter NW, Van Der Willigen C (1998) A mathematical and statistical analysis of the curves illustrating vulnerability of xylem to cavitation. Tree Physiol 18: 589-593

Pelloux JRM, Rusterucci C, Mellerowicz EJ (2007) New insights into pectin methylesterase structure and function. Trends Plant Sci 12: 267-277

Pesacreta TC, Groom LH, Rials TG (2005) Atomic force microscopy of the intervessel pit membrane in the stem of Sapium sebiferum (Euphorbiaceae). IAWA J 26: 397-426

Pfaffl MW (2001) A new mathematical model for relative quantification in real-time RT-PCR. Nucleic Acids Res 29: e45

Plavcova L, Hacke UG (2011) Heterogeneous distribution of pectin epitopes and calcium in different pit types of four angiosperm species. New Phytol 192: 885-897

Schneider CA, Rasband WS, Eliceiri KW (2012) NIH image to Image): 25 years of image analysis. Nat Methods 9: $671-675$

Scholz A, Rabaey D, Stein A, Cochard H, Smets E, Jansen S (2013) The evolution and function of vessel and pit characters with respect to cavitation resistance across 10 Prunus species. Tree Physiol 33: 684-694

Schulte PJ, Hacke UG, Schoonmaker ALC (2015) Pit membrane structure is highly variable and accounts for a major resistance to water flow through tracheid pits in stems and roots of two boreal conifer species. New Phytol 208: 102-113

Siedlecka A, Wiklund S, M-AI P, Micheli F, Lesniewska J, Sethson I, Edlund U, Richard L, Sundberg BR, Mellerowicz EJ (2008) Pectin methyl esterase inhibits intrusive and symplastic cell growth in developing wood cells of populus. Plant Physiol 146: 554-565

Sperry JS, Hacke UG (2004) Analysis of circular bordered pit function - I. Angiosperm vessels with homogenous pit membranes. Am J Bot 91: 369-385

Steudle E (2001) The cohesion-tension mechanism and the acquisition of water by plant roots. Annu Rev Plant Physiol Plant Mol Biol 52: 847-875

Tixier A, Cochard H, Badel E, Dusotoit-Coucaud A, Jansen S, Herbette S (2013) Arabidopsis thaliana as a model species for xylem hydraulics: does size matter? J Exp Bot 64: 2295-2305

Tixier A, Herbette S, Jansen S, Capron M, Tordjeman P, Cochard H, Badel E (2014) Modelling the mechanical behaviour of pit membranes in bordered pits with respect to cavitation resistance in angiosperms. Ann Bot 114: $325-334$ 
Tyree MT (1997) The cohesion-tension theory of sap ascent. Current controversies. J Exp Bot 48:

1753-1765

Wheeler JK, Sperry JS, Hacke UG, Hoang N (2005) Inter-vessel pitting and cavitation in woody Rosaceae and other vesselled plants: a basis for a safety versus efficiency trade-off in xylem transport. Plant Cell Environ 28: $800-812$

Willats WG, McCartney L, Mackie W, Knox JP (2001) Pectin: cell biology and prospects for functional analysis. Plant Mol Biol 47: 9-27

Wolf S, Mouille G, Pelloux J (2009) Homogalacturonan methyl-esterification and plant development. Mol Plant 2: $851-860$
Zhang Y, Klepsch M, Jansen S (2017) Bordered pits in xylem of vesselless angiosperms and their possible misinterpretation as perforation plates. Plant Cell Environ 40: 2133-2146

\section{Supporting Information}

Additional Supporting Information may be found in the online version of this article:

Fig. S1. Vulnerability to cavitation of poplars overexpressing constitutively PtxtPL-27. 